\title{
Countermeasures and Suggestions for the Co- construction and Sharing of Cultural Information Resources in Chengdu and Chongqing Against the Background of Two-city Economic Circle
}

\author{
Aihua Shen ${ }^{1, *} \mathrm{Yu} \mathrm{Liu}{ }^{1}$ \\ ${ }^{1}$ University of Electronic Science and Technology of China, Chengdu, Sichuan, China \\ *Corresponding author. Email: $1144706491 @ q q . c o m$
}

\begin{abstract}
As "promoting the construction of the Chengdu-Chongqing two-city economic circle" was written into the government work report, the strategic positioning of the Chengdu-Chongqing region has also been further elevated to a national strategy. It is of great practical significance to explore the co-construction and sharing of cultural information resources in Chengdu-Chongqing region in the context of the "two-city economic circle". By discussing the new characteristics of Chengdu-Chongqing cultural exchanges and the problems faced by the co-construction and sharing of cultural information resources against the background of the "two-city economic circle", this article points out that to strengthen the collaboration and sharing of cultural information resources between Chengdu and Chongqing, it is necessary to explore and build a scientific and effective coordination management and benefit balancing mechanism, establish a standardized and unified standard system for the coconstruction and sharing of cultural information resources, build a platform for the co-construction and sharing of cultural information resources between Chengdu and Chongqing, and strengthen the talent guarantee for the co-construction and sharing of cultural information resources.
\end{abstract}

Keywords: Two-city economic circle, Cultural exchange, Information resource sharing, Countermeasures and suggestions.

\section{INTRODUCTION}

In May 2020, the Third Session of the Thirteenth National People's Congress included "promoting the construction of the ChengduChongqing two-city economic circle" in the government work report, bringing new opportunities for the development of the ChengduChongqing region, and also proposing higher requirements. In the digital information age, the coconstruction and sharing of cultural information resources is of great significance to promote the development of society, economy and culture. In order to realize the exchange and cooperation in

*Fund: This paper is supported by the Phased Research Results of the 2020 Cultural Research Project "Chengdu and Chongqing Cultural Cities Exchange and Cooperation under the Background of the Two-City Economic Circle" by Propaganda Department of Chengdu Municipal Party Committee. cultural development between the long-term competing urban cultures in the context of the twocity economic circle, it is necessary to integrate the modern cultural resources of Chengdu and Chongqing, and strengthen the coordination and sharing of cultural information resources in Chengdu and Chongqing. The distinctive cultures and close historical origins of Chengdu and Chongqing have laid a good foundation for cultural exchanges and cooperation between the two regions. However, there are still problems in the coconstruction and sharing of cultural information resources between the two regions, such as lack of scientific coordination management and benefit balancing mechanism, lack of cultural information resource sharing platform, insufficient standardization, and insufficient talent guarantee. In the context of the "two-city economic circle", laying the foundation for Chengdu-Chongqing 
cultural exchanges and cooperation, integrating Chengdu-Chongqing modern cultural resources, and strengthening the coordination and sharing of cultural information resources between Chengdu and Chongqing are the inevitable requirements for promoting the formation of the development pattern of cultural exchanges in the two cities, and they are also inevitable choices made to conform to the trend of social development. Based on this, this article puts forward corresponding countermeasures and suggestions for the problems in the coconstruction and sharing of cultural information resources between Chengdu and Chongqing against the background of the "two-city economic circle".

\section{NEW FEATURES OF CHENGDU- CHONGQING CULTURAL EXCHANGES AND COOPERATION IN THE CONTEXT OF THE TWO-CITY ECONOMIC CIRCLE}

Chengdu and Chongqing, as the core cities of the two-city economic circle, are both regional cultural centers and economic growth centers. Economic growth lays the foundation for culture, and cultural development empowers the economy. In the context of the "two-city economic circle", the exchanges and cooperation between Chengdu and Chongqing have continued to develop in depth. Based on the original economic and trade cooperation, a new space for humanistic interaction has been further expanded, and cultural exchanges and cooperation have also shown a series of new characteristics.

\subsection{The Connotation of Exchanges and Cooperation Is Getting Richer and Richer}

The Chengdu-Chongqing two-city economic circle has become a national strategy. In this context, the cultural exchanges and cooperation between Chengdu and Chongqing are also developing in the direction of normalized exchanges and cooperation with higher standards and richer connotations. On the one hand, the depth of cooperation in Chengdu-Chongqing cultural exchanges has continued to increase; on the other hand, the areas and coverage of cooperation have continued to expand, and the carriers of ChengduChongqing cultural exchanges have also been continuously enriched. After the "two-city economic circle" strategy was proposed, Both
Sichuan and Chongqing Federation of Literary and Art reached a strategic cooperation framework agreement to deepen literary and artistic cooperation; the two regions will also work together to build the Bashu Cultural Tourism Corridor, and further promote cultural and tourism exchanges and cooperation with the Bashu culture of the same origin as the link. In addition, in order to further deepen exchanges and cooperation, the two regions have established the ChengduChongqing Two-city Economic Circle Innovation and Entrepreneurship Alliance and the University Alliance; they have also established many cooperative organizations such as the Cultural and Tourism Industry-Education Alliance of ChengduChongqing Two-city Economic Circle, and the Local Undergraduate University Talent Coordinated Development Center. ChengduChongqing has improved the depth of cooperation in all aspects such as cultural industry, cultural tourism cooperation, literature and art cooperation, talent and technical cooperation, etc., expanded the forms of cultural exchanges and cooperation between the two regions, and continuously enriched the new connotation of Chengdu-Chongqing cultural exchanges and cooperation.

\subsection{The Degree of Integration Is Gradually Increasing}

At present, under the new national strategic positioning of the Chengdu-Chongqing urban agglomeration to build a "Chengdu-Chongqing two-city economic circle", the exchanges and cooperation between Chengdu and Chongqing have gradually developed from the traditional perspective of focusing on the coordination and cooperation between the two to the overall perspective of an organic spatial interaction. While in terms of culture, it shows the characteristics of increasing integration of cultural exchanges. The geographical locations of the two regions are similar, and their cultures come down in one continuous line. Taking advantage of the "economic circle", on the basis of the continuous development of the transportation integration process between the two regions, the "cross-border" layout of cultural-related industries, the joint construction of cultural facilities, and the sharing of cultural and tourism markets are all constantly developing. The degree of integrated exchanges and cooperation of "I am in you and you are in me" has been continuously improved. 


\subsection{The Level of Digital Support Continues to Improve}

With the continuous improvement of the digital level of cultural construction in Chengdu and Chongqing, in terms of public cultural services, both regions have built digital platforms using "Internet + " and big data technologies. Driven by the economy, the informationization of cultural industries in both regions has also made rapid progress. In the process of exchanges and cooperation between the two regions, the support of digital information plays an important role, which plays an irreplaceable role in improving the efficiency and quality of cultural exchanges and cooperation, increasing the influence of cooperative projects, and promoting the development of indepth cultural exchanges and cooperation. On the basis of accelerating the economic development of the Chengdu and Chongqing, the exchanges and cooperation between the two regions continue to develop in depth, and the frequency of cultural information exchanges is also constantly increasing.

\section{THE PROBLEMS FACED BY CHENGDU-CHONGQING CULTURAL INFORMATION RESOURCE SHARING}

The coordination and sharing of information resources in regional cultural exchanges and cooperation is often of great significance. Supported by the continuous improvement of the digital level of cultural construction in the two regions, as the connotation of cultural cooperation and exchange between Chengdu and Chongqing is increasingly richer and the degree of integration is gradually improving, the demand for cultural information resource sharing in the exchange and cooperation between the two regions is also constantly increasing. However, the current level of information collaboration between Chengdu and Chongqing still can't meet the needs of long-term cooperation and development between the two regions, and the sharing of cultural information resources between the two regions still faces many problems.

\subsection{Lacking an Interconnected Cultural Information Resource Sharing Platform}

Both Chengdu and Chongqing have their own platforms for the construction and sharing of cultural information resources in some cultural fields. For example, in terms of public cultural service platforms, under the leadership of the Chongqing Cultural Commission, Chongqing has integrated its cultural resources and created the "CLOUD OF BA-YU CULTURE", a public cultural service platform that integrates information on cultural resources such as cultural activities, cultural venues, and cultural tourism; some areas of Chengdu also rely on corresponding technological advantages to build an information sharing platform. However, these platforms are only confined within their respective administrative regions, and belong to different systems, and the cultural fields involved and the cultural information resources included are also very limited. A unified digital platform that can realize the interconnection and intercommunication of cultural information resources between Chengdu and Chongqing has not yet been formed. In addition, under the call of the National Cultural Information Resource Sharing Project, Sichuan and Chongqing have established their own digital resource databases with local characteristics. However, due to the different affiliation and management systems of these resource banks in construction, they lack overall planning and unified coordination, and they often work in their own ways. There is a lack of unified standards and horizontal connections, and information barriers are obvious [1]. Therefore, it is difficult to achieve cross-database retrieval and coconstruction and sharing of information resources, which brings great difficulties to the development of regional joint cultural information resource cataloging and resource ordering and also hinders the realization of all-round and multi-field cultural exchanges and in-depth cooperation. Therefore, it is difficult to improve the quality and efficiency of the co-construction and sharing of Chengdu-Chongqing cultural information resources.

\subsection{Lacking Scientific Coordination Management Mechanism and Benefit Balancing Mechanism}

The exchange and sharing of cultural information resources across administrative regions involves multiple sharing subjects, and the diversification and non-subordination of sharing subjects will result in the differences in the goals and interests of the sharing subjects and the division of sharing systems and mechanisms. Authenticity and timeliness are the prerequisites for information to be shared, and in order to update system information in a timely manner, it needs to 
be driven by interests as a guarantee [2]. Chengdu and Chongqing has not yet established a unified management system mechanism for the coconstruction and sharing of cultural information resources, and it still lacks a scientific interestbalancing mechanism, which makes it easy to lead to separate management, repeated construction of cultural information resources, and untimely updates, etc. and as a result, cultural information resources are not fully and reasonably used and seriously wasted, and it is difficult to form a substantial sharing, which restricts the pace of their cultural exchanges and development.

\subsection{The Degree of Standardization and Normalization of the Co-construction and Sharing of Cultural Information Resources Is Insufficient}

To realize the co-construction and sharing of cultural information resources, standardization and normalization are the prerequisites. The lack of unified standards and norms will bring greater difficulties to the sharing of cultural information resources. On the one hand, different organizations will form differentiated metadata standards in the long-term development, making it difficult for information resources to circulate. Currently, there are obvious differences in heterogeneous data among public cultural institutions, which has become an important problem in the integration of public digital cultural resources [3]. On the other hand, the system and mechanism with clear boundaries between regions can also lead to huge differences in technical standards, network protocols, and system specifications. At present, in the process of constructing cultural information resources in Chengdu and Chongqing, the modes are different and independent, and as a result, the quality of the cultural information resource databases in the two regions is uneven, the data conflict with each other, and the coordination of information system is poor, which is not conducive to the sharing of cultural information resources.

\subsection{Insufficient Talent Guarantee for the Co-construction and Sharing of Cultural Information Resources}

High-quality inter-disciplinary talents are the key to promoting the efficient development of cultural information resource sharing. In an electronic, networked, and international environment, the sharing of cultural information resources requires a high-quality "interdisciplinary" talent team. The operation, use, and development of all kinds of high-grade, precise and advanced information technologies in resource sharing require professional talents, and people who lack relevant professional knowledge are unable to do this job. At present, the training of relevant talents is still relatively lacking, and the talent reserve is difficult to guarantee. In particular, there is a lack of a group of high-level personnel with certain professional knowledge and comprehensive capabilities, which also brings certain difficulties to the co-construction and sharing of cultural information resources.

\section{COUNTERMEASURES AND SUGGESTIONS FOR THE CO- CONSTRUCTION AND SHARING OF CHENGDU-CHONGQING CULTURAL INFORMATION RESOURCES}

In response to the problems faced by ChengduChongqing cultural information resource sharing, combined with the national strategy of "promoting the construction of the Chengdu-Chongqing twocity economic circle" and the new characteristics of Chengdu-Chongqing cultural exchanges and cooperation against the background of "two-city economic circle", the author puts forward countermeasures and suggestions to effectively promote the co-construction and sharing of Chengdu-Chongqing cultural information resources from the following four aspects.

\subsection{Cooperating to Build a Platform for the Co-construction and Sharing of Cultural Information Resources Between Chengdu and Chongqing}

Chengdu and Chongqing can make full use of the opportunities of integrated innovation and development to jointly build a cultural information resource sharing platform that can serve the two regions, or rely on the current public cultural service platforms that have been formed in the two regions to cooperate and upgrade. With the support of technologies such as "Internet + " and big data, the two regions can cooperate to create a "Cloud of Cheng-Yu Culture", integrate and share resources such as cultural activities and cultural tourism information between the two regions, and enhance the level of regional cultural information integration. At the same time, in order to avoid the 
outflow of secret-related information from government departments and realize the full sharing of cultural information resources for the people of the two regions, the two regions can split data at the front end of the platform according to the differences in the scope, degree and method of sharing [4]. In addition, the two regions can also rely on this information platform to promote the latest cultural tourism reciprocity policies, activities, and the newly developed cultural IP jointly developed by the two regions, and combine them with offline cultural tourism activities so as to expand the influence of cultural activities and cultural tourism projects in the two regions, paving the way for the cultivation of a common cultural brand between the two regions.

\subsection{Exploring and Building a Scientific and Effective Coordination Management and Benefit Balancing Mechanism}

In order to improve the quality and effectiveness of cultural exchanges and cooperation, Chengdu and Chongqing need to build a scientific and effective coordination management mechanism and strengthen the unified management and overall planning of cultural information resources. Chengdu and Chongqing need to reach agreement on the core elements of information sharing, strengthen the targeted services and connections of cultural information resources around the major cultural exchanges and cooperation projects between the two regions, and cooperate in data guidance and coordination to promote the integration and application of cultural information resources in the linkage work of cultural cooperation between the two regions.

In addition, an effective benefit balancing mechanism must be established. In order to ensure the equitable distribution of benefits, the two regions must adhere to the principles of fairness, risk sharing, and overall benefit maximization, and establish a unified intergovernmental interest coordination system within the region to achieve a harmonious relationship between stakeholders and objects [5]. At the same time, the two regions should provide certain compensation to the places and departments that have increased costs due to the openness and sharing of information, and provide appropriate incentives to the entities that actively participate in the co-construction and sharing, so as to realize the balance of interests among the sharing subjects, thereby promoting the sustainable development of the co-construction and sharing of regional cultural information resources.

\subsection{Establishing a Standardized and Unified Standard System for the Co- construction and Sharing of Cultural Information Resources}

The main feature of cross-regional information sharing is the heterogeneity between different autonomous systems. Due to the lack of consistent standards and specifications for data structure, format, and coding methods, it is difficult to achieve direct sharing [6]. Therefore, to promote the sharing of cultural information resources across administrative regions, it is necessary to establish a unified cultural information resource sharing standard system. The relevant functional departments of the two regions should cooperate with each other to jointly formulate and unify the relevant agreements, formats and other standards and regulations for the construction of cultural information resources, and use technical means to manage and supervise the entire process of access, invocation and use of information resources shared on the platform [7]. Through the establishment of a unified cultural information resource coconstruction and sharing standard system, the cultural information resources of the two regions can be collected, processed, handled with, evaluated, and exchanged under unified standards and regulations, solving the problem of inconsistent cultural information resource sharing standards between the two regions, and further laying the foundation for the co-construction and sharing of cultural information resources between the two regions.

\subsection{Strengthening the Talent Team Construction for the Co-construction and Sharing of Cultural Information Resources}

On the one hand, it is necessary to adhere to the equal emphasis on training and introduction. The two regions can cooperate in the development of personnel training and learning mechanisms. For most staff, they can have basic information technology expertise and work capabilities through comprehensive training. At the same time, the two regions should strengthen the construction of innovative talent teams. In addition to training, the two regions can also implement a proactive policy for the introduction of leading talents, take project 
needs as the guidance, and carry out targeted collaborative training, introduction and evaluation of talents in the region. On the other hand, it is necessary to establish a scientific performance appraisal mechanism, conduct dynamic assessments of talents on the basis of coordinated improvement of appraisal indicators, and formulate scientific incentive policies, so as to improve the enthusiasm and innovation ability of the talent team, and reduce the technical dependence and information security problems caused by the loss of technical personnel.

\section{CONCLUSION}

Under the new national strategic positioning of building the "Chengdu-Chongqing two-city economic circle", Chengdu-Chongqing cultural exchanges and cooperation are moving towards a higher level and richer connotation of normalized exchanges and cooperation. The degree of integrated exchanges and cooperation of "I am in you and you are in me" has been continuously improved. Supported by the continuous improvement of the digital level of cultural construction, the demand for cultural information resources sharing between the two regions is also increasing. However, the current level of information collaboration between Chengdu and Chongqing still can't meet the needs of long-term cooperation and development between the two regions, which limits the realization of the longterm sustainable cultural exchange and cooperation development goals of the two regions.

By building a platform for the co-construction and sharing of cultural information resources between Chengdu and Chongqing, a coordinated management and benefit balancing mechanism for co-construction and sharing of cultural information resources between the two regions can be promoted to promote the sustainable development of coconstruction and sharing of cultural information resources in Chengdu and Chongqing. In addition, the establishment of a unified cultural information resource co-construction and sharing standard system can enable the collection, processing, handling, evaluation, and exchange of cultural information resources in the two regions to be carried out under unified standards and regulations. Strengthening the training, introduction and evaluation of informatization talents can promote the realization of the collaboration and sharing of cultural information resources in Chengdu and Chongqing. Improving the continuity and efficiency of information exchange in cultural cooperation between the two regions can further improve the quality and effectiveness of cultural exchanges and cooperation between the two cities, and promote the in-depth development of cultural exchanges and cooperation between the two regions.

\section{AUTHORS' CONTRIBUTIONS}

Aihua Shen is responsible for the analysis and writing. $\mathrm{Yu}$ Liu contributed to instructing and revising.

\section{REFERENCES}

[1] Wu Gao. Investigation and Thoughts on the Status Quo of the Construction of Local Public Digital Cultural Characteristic Resources - Taking the Construction of Characteristic Digital Resources of the Provincial Branch of the National Cultural Information Resource Sharing Project as an Example [J]. Library Development, 2016, (1): 39-46. (in Chinese)

[2] Shen Quhe. The Legal Regulation of Government Information Sharing in China And a Comment on the "Interim Measures for the Administration of Government Information Resource Sharing"[J]. New Vision, 2017,(1):55-60. (in Chinese)

[3] Xiao Ximing, Liu Qiaoyuan. Public Digital Cultural Resources Integration Based on Metadata Repository [J]. Library, 2015,(9):1721,26. (in Chinese)

[4] Zhou Ying. Research on the Existing Problems and Countermeasures of Information Sharing Among Government Departments Based on the Investigation and Analysis of City A [J]. Nan Fang Lun Kan, 2019(08):5256. (in Chinese)

[5] Zhang Xin, Wnag Zhiyuan, Zhu Chunyan, et al. Local Government Game Behavior and Government Role Positioning in Regional Economic Integration $[\mathrm{J}]$. Journal of Commercial Economics, 2017, (6): 119-122. (in Chinese)

[6] Zou Hongxia, Wang Zhen, $\mathrm{Hu}$ Xinjie, et al. Cross Domain Information Sharing Application Based On Standardized Modeling [J]. Computer Applications and Software, 2019,36(2):29-32,38. 
DOI:10.3969/j.issn.1000-386x.2019.02.006.

(in Chinese)

[7] Song Yi, An Xiaomi, Fan Lingjun, Ma Guanghui. Collaborative Mechanism of Government Information Resources Sharing in Big Data Era: a Case Study of Haishu District, Ningbo City in China [J]. Information Studies: Theory \& Application, 2018, 41(06):64-69. (in Chinese) 